\title{
Powerfully nilpotent groups of maximal powerful class
}

\author{
G. Traustason ${ }^{1} \cdot$ J. Williams ${ }^{1}$
}

Received: 12 June 2019 / Accepted: 9 September 2019 / Published online: 13 September 2019 (c) The Author(s) 2019

\begin{abstract}
In this paper we continue the study of powerfully nilpotent groups started in Traustason and Williams (J Algebra 522:80-100, 2019). These are powerful $p$-groups possessing a central series of a special kind. To each such group one can attach a powerful class that leads naturally to the notion of a powerful coclass and classification in terms of an ancestry tree. The focus here is on powerfully nilpotent groups of maximal powerful class but these can be seen as the analogs of groups of maximal class in the class of all finite $p$-groups. We show that for any given positive integer $r$ and prime $p>r$, there exists a powerfully nilpotent group of maximal powerful class and we analyse the structure of these groups. The construction uses the Lazard correspondence and thus we construct first a powerfully nilpotent Lie ring of maximal powerful class and then lift this to a corresponding group of maximal powerful class. We also develop the theory of powerfully nilpotent Lie rings that is analogous to the theory of powerfully nilpotent groups.
\end{abstract}

Keywords Powerful $\cdot$ p-group $\cdot$ Nilpotent $\cdot$ p-group $\cdot$ Coclass $\cdot$ Maximal class

Mathematics Subject Classification 20D15 · 20F40

\section{Introduction}

In this paper we continue the study of powerfully nilpotent $p$-groups started in [6] and continued in [7]. Powerful $p$-groups were introduced by Lubotzky and Mann in [5]. The class of powerfully nilpotent groups is a special subclass of these, containing groups that possess a central series of a special kind. We start by recalling the basic terms. Let $G$ be a finite $p$-group where $p$ is a prime.

\section{Communicated by A. Constantin.}

\footnotetext{
$凶$ G. Traustason gt223@bath.ac.uk

1 Department of Mathematical Sciences, University of Bath, Bath, UK
} 
Definition Let $H \leq K \leq G$. An ascending chain of subgroups

$$
H=H_{0} \leq H_{1} \leq \cdots \leq H_{n}=K
$$

is powerfully central in $G$ if $\left[H_{i}, G\right] \leq H_{i-1}^{p}$ for $i=1, \ldots, n$. Here $n$ is called the length of the chain.

Definition A powerful p-group $G$ is powerfully nilpotent if it has an ascending chain of subgroups of the form

$$
\{1\}=H_{0} \leq H_{1} \leq \cdots \leq H_{n}=G
$$

that is powerfully central in $G$.

Definition If $G$ is powerfully nilpotent then the powerful nilpotence class of $G$ is the shortest length that a powerfully central chain of $G$ can have.

Definition We say that a finite $p$-group $G$ is strongly powerful if $[G, G] \leq G^{p^{2}}$.

In [6] we showed that a strongly powerful $p$-group is powerfully nilpotent of powerful class at most $e-1$ where $p^{e}$ is the exponent of the group.

The upper powerfully central series. This is defined recursively as follows: $\hat{Z}_{0}(G)=$ $\{1\}$ and for $n \geq 1$,

$$
\hat{Z}_{n}(G)=\left\{a \in G:[a, x] \in \hat{Z}_{n-1}(G)^{p} \text { for all } x \in G\right\} .
$$

Notice in particular that $\hat{Z}_{1}(G)=Z(G)$.

Definition Let $G$ be a powerfully nilpotent $p$-group of powerful class $c$ and order $p^{n}$. We define the powerful coclass of $G$ to be the number $n-c$.

A natural approach is to develop something that corresponds to a coclass theory for finite $p$-groups where coclass is replaced by powerful coclass and in [6] we proved that there are indeed, for any fixed prime $p$, finitely many powerfully nilpotent $p$-groups of any given powerful coclass. More precisely, if $G$ is a powerfully nilpotent $p$-group of rank $r$ and exponent $p^{e}$ then we showed that $r \leq n-c+1$ and $e \leq n-c+1$. This together with $n \leq r e$ gives the result.

The ancestry tree. Let $p$ be a fixed prime. The vertices of the ancestry tree are all the powerfully nilpotent $p$-groups (one for each isomorphism class). Two vertices $G$ and $H$ are joined by a directed edge from $H$ to $G$ if and only if $H \cong G / Z(G)^{p}$ and $G$ is not abelian. Notice that this implies that $Z(G)^{p} \neq\{1\}$ and thus the powerful class of $G$ is one more than that of $H$. We then also say that $G$ is a direct ancestor of $H$ or that $H$ is a direct descendant of $G$, and we write $H \rightarrow G$.

Let $c(H), c(G)$ be the powerfully nilpotent classes of $H$ and $G$ and let $d(H), d(G)$ be the powerful coclasses, that is $d(H)=n(H)-c(H)$ and $d(G)=n(G)-c(G)$ where the orders of $H$ and $G$ are $p^{n(H)}$ and $p^{n(G)}$. Notice that $c(H)=c(G)+1$ and that $d(H) \geq d(G)$ with equality if and only if $\left|Z(G)^{p}\right|=p$.

We will now recall some more terms from [6]. 
Definition Let $G$ be a powerfully nilpotent $p$-group and let $k$ be the largest nonnegative integer such that

$$
p=\left|Z(G)^{p}\right|=\left|\frac{\hat{Z}_{2}(G)^{p}}{\hat{Z}_{1}(G)^{p}}\right|=\cdots=\left|\frac{\hat{Z}_{k}(G)^{p}}{\hat{Z}_{k-1}(G)^{p}}\right| .
$$

we refer to $\hat{Z}_{k}(G)^{p}$ as the tail of $G$ and $k$ as the length of the tail.

Remark If $G$ has a tail of length $k$ then $G, G / \hat{Z}_{1}(G)^{p}, G / \hat{Z}_{2}(G)^{p}, \ldots, G / \hat{Z}_{k}(G)^{p}$ all have the same powerful coclass.

Definition Let $G$ be a powerfully nilpotent $p$-group. We say that $G$ has maximal tail if the tail of $G$ is $G^{p}$.

The following summarises some of the properties of groups with a maximal tail.

Theorem [6] Let $G$ be a powerfully nilpotent group of rank $r \geq 2$ that has a maximal tail. Suppose $G$ has order $p^{n}$, powerful class $c$ and exponent $p^{e}$. Let $t$ be the length of the tail.

(a) We have $c-1 \leq t \leq c$. It follows also that $n-c \leq r \leq n-c+1$.

(b) We have $t \leq 1+\frac{r(\bar{r}-1)}{2}$.

(c) We have $\operatorname{rank}(G)>\operatorname{rank}\left(G^{p}\right)>\cdots>\operatorname{rank}\left(G^{p^{e-2}}\right)$.

We conjectured in [6] that the bound given in (b) is attained and we will show in this paper that this is the case when $p>r$. A powerfully nilpotent group, for which this bound is attained, will be called a group of maximal powerful class.

The paper is organised as follows. In Sect. 2 we will develop the theory of powerfully nilpotent Lie rings that is analogous to the theory of powerfully nilpotent groups with similar results. In Sect. 3 we show the existence of a powerfully nilpotent Lie ring of maximal powerful class for any prime $p$ and any rank $r$ and we show that the structure of Lie rings of maximal powerful class is constrained. In the final section we then use the Lazard correspondence to obtain the analogous results for powerfully nilpotent $p$-groups of maximal powerful class when $p>r$. The main structure result, Theorem 4.2, shows some parallels with the structure of groups with maximal class (see for example [1] or Chapter 3.14 in [2]) in the class of all finite $p$-groups. We refer to [4] for an account of coclass theory for finite $p$-groups.

\section{Powerfully nilpotent Lie rings}

Definition Let $L$ be a finite Lie ring of $p$-power order and let $M, N$ be subrings where $M \leq N \leq L$. We say that an ascending chain of subrings

$$
M=J_{0} \leq J_{1} \leq \cdots \leq J_{n}=N
$$

is powerfully central in $L$ if $\left[J_{i}, L\right] \leq p J_{i-1}$ for $i=1, \ldots, n$. Here $n$ is called the length of the chain. 
Definition Let $L$ be a finite Lie ring of $p$-power order. We say that $L$ is powerfully nilpotent if it has an ascending chain of ideals of the form

$$
0=J_{0} \leq J_{1} \leq \cdots \leq J_{n}=L
$$

that is powerfully central in $L$.

Definition If $L$ is powerfully nilpotent then the powerful nilpotence class of $L$ is the shortest length that a powerfully central chain of $L$ can have.

Definition Let $L$ be a finite Lie ring of $p$-power order. We say that $L$ is powerful if $[L, L] \leq p L$ and strongly powerful if $[L, L] \leq p^{2} L$.

Remark Clearly a powerfully nilpotent Lie ring is powerful. The next lemma shows that a strongly powerful Lie ring is powerfully nilpotent.

Lemma 2.1 Let L be a strongly powerful Lie ring of characteristic $p^{e}$. Then $L$ is powerfully nilpotent. Furthermore the powerful class is at most $e-1$ if $e \geq 2$ and the powerful class is equal to $e$ if $e \leq 1$.

Proof The result for $e \leq 1$ is clear so we only need to consider the situation when $e \geq 2$. In order to deal with this case, it suffices to show that

$$
L>p L>p^{2} L>\cdots>p^{e-2} L>\{0\}
$$

is powerfully central. This follows from $\left[p^{e-2} L, L\right] \leq p^{e-2}\left(p^{2} L\right)=\{0\}$ and $\left[p^{k} L, L\right] \leq p^{k}\left(p^{2} L\right)=p\left(p^{k+1} L\right)$ when $0 \leq k<e-2$.

The upper powerfully central series. This is defined recursively as follows: $\hat{Z}_{0}(L)=$ $\{0\}$ and for $n \geq 1$,

$$
\hat{Z}_{n}(L)=\left\{a \in L:[a, x] \in p \hat{Z}_{n-1}(L) \text { for all } x \in L\right\} .
$$

Remark The upper powerfully central series is clearly the fastest ascending series that is powerfully central. It is easy to see that $L$ is powerfully nilpotent if and only if $L=\hat{Z}_{n}(L)$ for some $n$ and the smallest such $n$ is then the powerful class.

The next result is analogous to a corresponding result from [6].

Proposition 2.2 Let L be a finite Lie ring of characteristic $p^{e}$ where $e \geq 2$. If $L / p^{2} L$ is powerfully nilpotent, then $L$ is powerfully nilpotent. Furthermore if $L / p^{2} L$ has powerful class $m$, then the powerful class of $L$ is at most $(e-1) m$.

Proof Suppose

$$
L / p^{2} L=J_{0} / p^{2} L>J_{1} / p^{2} L>\cdots>J_{m-1} / p^{2} L>\{0\}
$$


is the upper powerfully central series for $L / p^{2} L$. As $L / p^{2} L$ is powerful, we have $[L, L] \leq p L+p^{2} L=p L$ and $L$ is powerful. Thus in particular $[p L, L]=p[L, L] \leq$ $p^{2} L$ and $p L \leq J_{m-1}$. Hence

$$
L=J_{0}>J_{1}>\cdots>J_{m-1}>J_{m}=p L
$$

is powerfully central in $L$. Consider the descending chain

$$
\begin{aligned}
& L \quad=J_{0} \geq J_{1} \quad \geq \cdots \geq J_{m} \quad=p L \\
& p L \quad=p J_{0} \geq p J_{1} \geq \cdots \geq p J_{m}=p^{2} L \\
& p^{e-2} L=p^{e-2} J_{0} \geq p^{e-2} J_{1} \geq \cdots \geq p^{e-2} J_{m}=p^{e-1} L .
\end{aligned}
$$

We know already that the first line gives us a powerfully central chain in $L$. Thus $\left[p^{k} J_{i}, L\right]=p^{k}\left[J_{i}, L\right] \leq p^{k+1} J_{i+1}$ for $0 \leq i \leq m-1$ and $0 \leq k \leq e-2$. It follows that we have a powerfully central chain in $L$. Notice also that $\left[p^{e-2} J_{m-1}, L\right]=$ $p^{e-2}\left(p^{2} L\right)=\{0\}$ and thus $p^{e-2} J_{m-1} \leq Z(L)$. It follows that $L$ is powerful with powerful class $(e-1) m$.

Like for powerfully nilpotent groups there is a natural classification of powerfully nilpotent Lie rings in terms of a powerful coclass and an ancestry tree.

Definition Let $L$ be a powerfully nilpotent Lie ring of powerful class $c$ and order $p^{n}$. We define the powerful coclass of $L$ to be the number $n-c$.

The ancestry tree. Let $p$ be a fixed prime. The vertices of the ancestry tree are all the powerfully nilpotent Lie rings of prime power order (one for each isomorphism class). Two vertices $L$ and $M$ are joined by a directed edge from $M$ to $L$ if and only if $M \cong L / p Z(L)$ and $L$ is not abelian. Notice that this implies that $p Z(L) \neq\{0\}$ and thus the powerful class of $L$ is one more than that of $M$. We then say that $L$ is a direct ancestor of $M$ or that $M$ is a direct descendant of $L$ and write $M \rightarrow L$.

Remark Suppose $M$ is a powerfully nilpotent Lie ring of order $p^{n(M)}$ and powerful class $c(M)$ and suppose that $L$ is a direct ancestor of $M$. Then $L$ has powerful class $c(M)+1$ and order $p^{n(L)}=|L / p Z(L)| \cdot|p Z(L)|=p^{n(H)+k}$ where $|p Z(L)|=p^{k}$. Thus the powerful coclass of $L$ is $d(L)=n(M)+k-(c(M)+1)=(n(M)-c(M))+$ $(k-1)$ and thus $d(L) \geq d(M)$ where $d(M)$ is the powerful class of $M$. Notice that we have equality if and only if $|p Z(L)|=p$.

Lemma 2.3 Let L be a powerfully nilpotent Lie ring of powerful class $c \geq 2$, then

$$
[L, L]=\left[\hat{Z}_{c}(L), L\right]>\left[\hat{Z}_{c-1}(L), L\right]>\cdots>\left[\hat{Z}_{1}(L), L\right]=\{0\}
$$

and

$$
p L=p \hat{Z}_{c}(L) \geq p \hat{Z}_{c-1}(L)>\cdots>p \hat{Z}_{1}(L)>p \hat{Z}_{0}(L)=\{0\} .
$$

In particular $|p L| \geq|[L, L]| \geq p^{c-1}$. 
Proof Suppose $2 \leq j \leq c$. If $\left[\hat{Z}_{j}(L), L\right]=\left[\hat{Z}_{j-1}(L), L\right]$, then $\left[\hat{Z}_{j}(L), L\right] \leq$ $p \hat{Z}_{j-2}(L)$ and thus we get the contradiction that $\hat{Z}_{j}(L) \leq \hat{Z}_{j-1}(L)$. The proof of the latter strict inequalities is similar. Let $1 \leq j \leq c-1$. If $p \hat{Z}_{j}(L)=p \hat{Z}_{j-1}(L)$ then $\left[\hat{Z}_{j+1}(L), L\right] \leq p \hat{Z}_{j-1}(L)$ and thus $\hat{Z}_{j+1}(L)=\hat{Z}_{j}(L)$ that gives the contradiction that the powerful class is at most $j \leq c-1$.

We are going to see that, as for powerfully nilpotent groups, there are for a fixed prime $p$ only finitely many powerfully nilpotent Lie rings of $p$-power order of any given coclass. This will follow from specific bounds for the rank and exponent in terms of the coclass. We start with the rank.

Proposition 2.4 Let $L$ be a powerfully nilpotent Lie ring of rank $r$, order $p^{n}$ and powerful class $c$. Then $r \leq n-c+1$.

Proof It follows from Lemma 2.3 that $p^{c-1} \leq|p L|=p^{n-r}$. The result follows.

In order to get the bound for the exponent in terms of the coclass we need first some more structure results for powerfully nilpotent Lie rings.

Let $L$ be any powerfully nilpotent Lie ring of characteristic $p^{e}$, order $p^{n}$ and rank $r$. Suppose

$$
L=J_{0}>J_{1}>\cdots>J_{m-1}>J_{m}=p L
$$

is the powerfully central chain as given in the proof of Proposition 2.2. We can always refine such a powerfully central chain to get a chain of length $r$ such that the factors are of order $p$. Without loss of generality we can thus assume that we have a powerfully central chain

$$
L=J_{0}>J_{1}>\cdots>J_{r}=p L
$$

such that $\left|J_{i} / p L\right|=p^{r-i}$ for $i=0, \ldots, r$. We thus have generators $a_{1}, \ldots, a_{r}$ for $L$ such that

$$
J_{i}=\mathbb{Z} a_{i+1}+\cdots+\mathbb{Z} a_{r}+p L=\mathbb{Z} p a_{1}+\cdots+\mathbb{Z} p a_{i}+\mathbb{Z} a_{i+1} \cdots+\mathbb{Z} a_{r} .
$$

We would like to choose $a_{1}, \ldots, a_{r}$ so that the choice best reflects the structure of the Lie ring $L$. As we saw in the proof of Proposition 2.2, we then get a powerfully central series

$$
\begin{aligned}
L & =J_{0} \geq J_{1} \geq \cdots \geq J_{r}=p L \\
p L & =p J_{0} \geq p J_{1} \geq \cdots \geq p J_{r}=p^{2} L \\
& \vdots \\
p^{e-2} L & =p^{e-2} J_{0} \geq p^{e-2} J_{1} \geq \cdots \geq p^{e-2} J_{r}=p^{e-1} L .
\end{aligned}
$$

For $0 \leq i \leq r-1$ we have $J_{i}=J_{i+1}+\mathbb{Z} a_{i+1}$. Notice that if $p J_{i}=p J_{i+1}$, then $p a_{i+1}=p b_{i}$ for some $b_{i} \in J_{i+1}$ and replacing $a_{i+1}$ by $a_{i+1}-b_{i}$, we can assume that $p a_{i+1}=0$ whenever $p J_{i}=p J_{i+1}$. Having done this we can move all the generators that are of order $p$ in front of the others (keeping the previous 
order unchanged otherwise) and still have that (1) gives us a powerfully central chain. We can thus assume that for some $0 \leq s \leq r$ we have $p a_{1}=\cdots=p a_{s}=0$ and that $p L=p J_{s}>p J_{s+1}>\cdots>p J_{r}=p^{2} L$. Notice that the rank of $p L$ is the number of generators of order at least $p^{2}$. We have $p L=0$ if $s=r$ and otherwise $0 \leq s<r$ and $\{0\}<p L=p J_{s}$. Suppose we are in the latter situation. In this case we have for $s \leq j \leq r-1$ that $p^{2} J_{i}=p^{2} J_{i+1}$ if and only if there exists $x \in J_{i} \backslash J_{i+1}$ such that $p^{2} x=0$. We can thus choose our generators such that furthermore $p^{2} J_{i}=p^{2} J_{i+1}$ if and only if $a_{i+1}$ has order $p^{2}$. Notice again that the rank of $p^{2} L$ is then the number of generators of order at least $p^{3}$. Continuing in this manner, considering next $p^{3} J_{0} \geq p^{3} J_{1} \geq \cdots \geq p^{3} J_{r}=p^{4} L$ and then the $p^{4}$ th powers and so on, we eventually arrive at a set of generators $a_{1}, \ldots, a_{r}$ with some specific properties. If for $0 \leq i \leq r$ we let $s(i)$ be the number of generators of order $p^{i}$ then $\left|p^{i-1} L / p^{i} L\right|=p^{s(i)+s(i+1)+\cdots+s(e)}$. Then

$$
\begin{aligned}
|L| & =|L / p L| \cdot\left|p L / p^{2} L\right| \cdots\left|p^{e-1} L / p^{e} L\right| \\
& =p^{s(1)+\cdots+s(e)} p^{s(2)+\cdots+s(e)} \cdots p^{s(e)} \\
& =p^{s(1)} p^{2 s(2)} \cdots p^{e s(e)} \\
& =o\left(a_{1}\right) \cdots o\left(a_{r}\right) .
\end{aligned}
$$

As $G=\mathbb{Z} a_{1}+\cdots+\mathbb{Z} a_{r}$, it follows that this sum is a direct sum of cyclic groups. We have thus shown:

Proposition 2.5 Let $L$ be a powerfully nilpotent Lie ring of rank $r$, characteristic $p^{e}$ and order $p^{n}$. Then we can choose generators for L as a Lie ring such that we get a direct sum of cyclic groups

$$
L=\mathbb{Z} a_{1} \oplus \cdots \oplus \mathbb{Z} a_{r}
$$

and where for $J_{i}=\mathbb{Z} p a_{1}+\cdots+\mathbb{Z} p a_{i}+\mathbb{Z} a_{i+1}+\cdots+\mathbb{Z} a_{r}$ we get a powerfully central chain

$$
\begin{aligned}
L & =J_{0} \geq J_{1} \\
p L & =p J_{0} \geq p J_{1} \geq \cdots \geq J_{r}=p L \\
& \geq \\
& \vdots \\
p^{e-2} L & =p^{e-2} J_{0} \geq p^{e-2} J_{1} \geq \cdots \geq p^{2} L
\end{aligned}
$$

Furthermore we can assume that for some $0 \leq s \leq r, o\left(a_{1}\right)=\cdots=o\left(a_{s}\right)=p$ and $o\left(a_{s+1}\right), \ldots, o\left(a_{r}\right)>p$.

Remark If we omit repetitions in the chain above, then all the factors have order $p$.

Lemma 2.6 Let $L$ be a powerful Lie ring of p-power order. If $p^{k} L$ is a cyclic group, then $p^{k} L \leq Z(L)$.

Proof Suppose $p^{k} L=\mathbb{Z} p^{k} c$. Then $\left[p^{k} L, L\right]=\left[p^{k} c, L\right]=\left[c, p^{k} L\right]=\mathbb{Z}\left[c, p^{k} c\right]=$ $\{0\}$. 
Lemma 2.7 Let L be a powerful Lie ring of p-power order and suppose that $p^{k} L$ has rank $s \geq 2$. Let $a_{1}, \ldots, a_{r}$ be as in Proposition 2.5 and that $p^{k} L=\mathbb{Z} p^{k} a_{i_{1}}+\cdots+$ $\mathbb{Z} p^{k} a_{i_{s}}$ with $i_{1}<i_{2}<\cdots<i_{s}$. Then the chain

$$
p^{k} L=p^{k} \mathbb{Z} a_{i_{1}}+\cdots+p^{k} \mathbb{Z} a_{i_{s}}>p^{k} \mathbb{Z} a_{i_{3}}+\cdots+p^{k} \mathbb{Z} a_{i_{s}}
$$

is powerfully central in $L$.

Proof We know from Proposition 2.5 that

$$
p^{k} \mathbb{Z} a_{i_{2}}+\cdots+p^{k} \mathbb{Z} a_{i_{s}}>p^{k} \mathbb{Z} a_{i_{3}}+\cdots+p^{k} \mathbb{Z} a_{i_{s}}
$$

is powerfully central in $L$. Then, using the fact that $\left[a_{i_{1}}, a_{i_{1}}\right]=0$,

$$
\begin{aligned}
{\left[p^{k} L, L\right] } & =\left[p^{k} \mathbb{Z} a_{i_{1}}+\cdots+p^{k} \mathbb{Z} a_{i_{s}}, L\right] \\
& =\left[\mathbb{Z} a_{i_{1}}+\cdots+\mathbb{Z} a_{i_{s}}, p^{k} L\right] \\
& =p^{k}\left[\mathbb{Z} a_{i_{1}}+\cdots+\mathbb{Z} a_{i_{s}}, \mathbb{Z} a_{i_{1}}+\cdots+\mathbb{Z} a_{i_{s}}\right] \\
& =p^{k}\left[\mathbb{Z} a_{i_{2}}+\cdots+\mathbb{Z} a_{i_{s}}, \mathbb{Z} a_{i_{1}}+\cdots+\mathbb{Z} a_{i_{s}}\right] \\
& =\left[\mathbb{Z} a_{i_{2}}+\cdots+\mathbb{Z} a_{i_{s}}, p^{k} \mathbb{Z} a_{i_{1}}+\cdots+p^{k} \mathbb{Z} a_{i_{s}}\right] \\
& =\left[\mathbb{Z} a_{i_{2}}+\cdots+\mathbb{Z} a_{i_{s}}, p^{k} L\right] \\
& =\left[p^{k} \mathbb{Z} a_{i_{2}}+\cdots+p^{k} \mathbb{Z} a_{i_{s}}, L\right] \\
& \leq p\left(p^{k} \mathbb{Z} a_{i_{3}}+\cdots+p^{k} \mathbb{Z} a_{i_{s}}\right) .
\end{aligned}
$$

This finishes the proof.

Remark This lemma tells us that if we omit repetitions in the powerfully central chain from Proposition 2.5 then the second term in each line (where the rank of $p^{k} L$ is $s \geq 2$ )

$p^{k} L=p^{k} \mathbb{Z} a_{i_{1}}+\cdots+p^{k} \mathbb{Z} a_{i_{s}}>p^{k} \mathbb{Z} a_{i_{2}}+\cdots+p^{k} \mathbb{Z} a_{i_{s}}>\cdots>p^{k} \mathbb{Z} a_{i_{s}}>p^{k+1} L$

can also be omitted and we still have a powerfully central chain.

Theorem 2.8 Let L be a powerfully nilpotent Lie ring of order $p^{n}$, powerful class $c$ and characteristic $p^{e}$. Then $e \leq n-c+1$.

Proof This is easy to see when $L$ is of rank 1 so we can assume that the rank of $L$ is at least 2. Let $k$ be the largest non-negative integer such that the rank of $p^{k} L$ is greater than or equal to 2 . Let $r_{i}$ be the rank of $p^{i} L$ for $i=0,1, \ldots, k$ and let $p^{n_{0}}=\left|p^{k+1} L\right|$. Notice then that

$$
\begin{aligned}
& e=k+1+n_{0} \\
& n=r_{0}+r_{1}+\cdots+r_{k}+n_{0} .
\end{aligned}
$$


By Lemma 2.7 there extis for each $0 \leq j \leq k$ a descending chain

$$
p^{j} L=I_{0}>I_{1}>\cdots>I_{r_{j}-1}=p^{j+1} L
$$

that is powerfully central in $L$. Adding up for $j=0,1, \ldots, k$ and using the fact from Lemma 2.6 that $p^{k+1} L \leq Z(L)$, we get a central chain for $L$ that is of length $\left(r_{0}-1\right)+\left(r_{1}-1\right)+\cdots+\left(r_{k}-1\right)+1$. Hence $c \leq\left(r_{0}-1\right)+\left(r_{1}-1\right)+\cdots+\left(r_{k}-1\right)+1$ and we conclude that

$$
\begin{aligned}
n-c & \geq n-\left[\left(r_{0}-1\right)+\cdots+\left(r_{k}-1\right)+1\right] \\
& =\left(r_{0}+\cdots+r_{k}+n_{0}\right)-\left[\left(r_{0}-1\right)+\cdots+\left(r_{k}-1\right)+1\right] \\
& =k+n_{0} \\
& =e-1 .
\end{aligned}
$$

Hence $e \leq n-c+1$.

As a corollary we get a theorem that is analogous to one of the main results from [6]

Theorem 2.9 For each prime $p$ and non-negative integer $d$, there are only finitely many powerfully nilpotent Lie rings of p-power order that have powerful coclass $d$.

Proof Let $L$ be a powerfully nilpotent Lie ring of order $p^{n}$, rank $r$ and exponent $p^{e}$. Then $n \leq r e \leq(d+1)(d+1)$ and thus the order of $L$ is bounded by the coclass.

\section{Powerfully nilpotent Lie rings of maximal powerful class}

Definition Let $L$ be a powerfully nilpotent Lie ring of $p$-power order and let $k$ be the largest non-negative integer such that

$$
p=|p Z(L)|=\left|\frac{p \hat{Z}_{2}(L)}{p \hat{Z}_{1}(L)}\right|=\cdots=\left|\frac{p \hat{Z}_{k}(L)}{p \hat{Z}_{k-1}(L)}\right| .
$$

We refer to $p \hat{Z}_{k}(L)$ as the tail of $L$ and $k$ as the length of the tail.

Remark If $L$ has a tail of length $k$, then $L, L / p \hat{Z}_{1}(L), L / p \hat{Z}_{2}(L), \ldots, L / p \hat{Z}_{k}(L)$ all have the same powerful coclass.

Lemma 3.1 Rewrite the chain from Proposition 2.5 in ascending order without repetitions. Then suppose the chain up to and including $p L$ is

$$
\{0\}=M_{0}<M_{1}<\cdots<M_{t}=p L .
$$

We have that $M_{j} \leq p \hat{Z}_{j}(L)$ for $j=0, \ldots, t$. Also if the tail of $L$ is $p \hat{Z}_{k}(L)$, then $M_{i}=p \hat{Z}_{i}(L)$ for $i=0, \ldots, k$. 
Proof We prove the first part by induction on $0 \leq j \leq t$. This is obvious when $j=0$. Now suppose that $j \geq 1$ and that the result holds for smaller values of $j$. Let $q$ be the largest and then, for that $q, i$ be the largest such that $M_{j}=p^{q} J_{i}$. Then $0 \leq i \leq r-1$ and $p^{q} J_{i+1}=M_{j-1}$. Thus

$$
\left[p^{q-1} J_{i}, L\right] \leq p\left(p^{q-1} J_{i+1}\right)=M_{j-1} \leq p \hat{Z}_{j-1}
$$

by the induction hypothesis. Hence $p^{q-1} J_{i} \leq \hat{Z}_{j}(L)$ and thus $M_{j}=p^{q} J_{i} \leq p \hat{Z}_{j}(L)$. This finishes the inductive proof. The 2 nd part follows from the 1 st part and the fact that $\left|p \hat{Z}_{i}(L)\right|=p^{i}$ for $i=0, \ldots, k$.

Definition We say that a powerfully nilpotent Lie ring $L$ has maximal tail if the tail of $L$ is $p L$.

Remark If $L$ is abelian then $L$ has maximal tail if and only if $|p L|=p$ that happens if and only if

$$
L=\mathbb{Z} a_{1}+\cdots+\mathbb{Z} a_{r}+\mathbb{Z} b
$$

where $r \geq 0, o\left(a_{1}\right)=\cdots=o\left(a_{r}\right)=p$ and $o(b)=p^{2}$. In particular $L=\mathbb{Z} b$, with $o(b)=p^{2}$, is the only abelian Lie ring of $p$-power order that has rank 1 and maximal tail.

Theorem 3.2 Let L be a powerfully nilpotent Lie ring of rank $r \geq 2$ that has maximal tail. Suppose that L has order $p^{n}$, powerful class $c$ and characteristic $p^{e}$. Let $t$ be the length of the tail.

(a) We have $\operatorname{rank}(L)>\operatorname{rank}(p L)>\cdots>\operatorname{rank}\left(p^{e-2} L\right)$.

(b) We have that $c-1 \leq t \leq c$.

(c) We have $t \leq 1+\frac{r(r-1)}{2}$.

Proof (a) For $e \leq 2$ there is nothing to prove and we can thus assume that $e \geq 3$. Notice first that the rank of $p^{e-3} L$ must be at least 2. Otherwise, by Lemma 2.6, we would have that $p^{e-3} L \leq Z(L)$ and thus $p^{e-2} L \leq p Z(L)$. As $|p Z(L)|=p$ we would then get the contradiction that $p^{e-1} L=\{0\}$. Thus $L, p L, \ldots, p^{e-3} L$ all have rank at least 2 . Let $0 \leq k \leq e-3$ and consider the chain from Proposition 2.5. The subchain

$$
p^{k} L=p^{k} J_{0} \geq p^{k} J_{1} \geq \cdots \geq p^{k} J_{r}=p^{k+1} L
$$

is powerfully central in $L$. Suppose that the rank of $p^{k} L$ is $s \geq 2$. Omitting repetitions we get a chain

$$
\begin{aligned}
p^{k} L= & \mathbb{Z} p^{k} a_{i_{1}}+\cdots+\mathbb{Z} p^{k} a_{i_{s}}+p^{k+1} L>\mathbb{Z} p^{k} a_{i_{2}}+\cdots+\mathbb{Z} p^{k} a_{i_{s}}+p^{k+1} L \\
& >\cdots>\mathbb{Z} p^{k} a_{i_{s}}+p^{k+1} L>p^{k+1} L
\end{aligned}
$$

with $1 \leq i_{1}<i_{2}<\cdots<i_{s} \leq r$. We know from Lemma 2.7 that we can omit the 2 nd term and still have a powerfully central chain. Let $E=p^{k} L$ and 
$F=\mathbb{Z} p^{k} a_{i_{3}}+\cdots+\mathbb{Z} p^{k} a_{i_{s}}+p^{k+1} L$. We know from Lemma 3.1 that $p F=$ $p \hat{Z}_{l}(L)$ for some $0 \leq l \leq t$. As $[E, L] \leq p F$, it follows that $E \leq \hat{Z}_{l+1}(L)$ and thus $p E \leq p \hat{Z}_{l+1}(\bar{L})$. We show that $[p E: p F] \leq p$. This is of course clear if $p E=p F$. Otherwise $p \hat{Z}_{l+1}(L) \geq p E>p F=p \hat{Z}_{l}(L)$ and thus we know from Lemma 3.1 that $[p E: p F]=\left[p \hat{Z}_{l+1}(L): p \hat{Z}_{l}(L)\right]=p$. Thus $[p E: p F]$ is either 1 or $p$ and thus the rank of $p^{k+1} L$ is at most $s-1$.

(b) By Lemma 2.3 we know that $p \hat{Z}_{c-2}(L)<p \hat{Z}_{c-1}(L) \leq p \hat{Z}_{c}(L)=p L$. Thus $c-1 \leq t \leq c$.

(c) By part (a) the largest potential tail occurs when $\operatorname{rank}(L)=r, \operatorname{rank}(p L)=$ $r-1, \ldots, \operatorname{rank}\left(p^{r-1} L\right)=1$. By Lemma 2.6 we then have $p^{r-1} L \leq Z(G)$ and thus $p^{r} L \leq p Z(G)$. For there to be a tail of length greater than 0 we need $|p Z(L)|=p$ and then $\left|p^{r} L\right| \leq p$. The tail can't thus be larger than

$|p L|=\left|p L / p^{2} L\right| \cdot\left|p^{2} L / p^{3} L\right| \cdots\left|p^{r-2} L / p^{r-1} L\right| \cdot\left|p^{r-1} L\right|=p^{(r-1)+\cdots+2+2}$.

This finishes the proof.

Remark We know from Theorem 3.2 that if $L$ has rank $r$ and maximal tail, then the length of the tail is at most $t=1+r(r-1) / 2$. We have also seen that in order for this upperbound to be attained we would need $\operatorname{rank}(L)=r, \operatorname{rank}(p L)=$ $r-1, \ldots, \operatorname{rank}\left(p^{r-2} L\right)=2$ and that $p^{r-1} L$ is of rank 1 and order $p^{2}$. In particular, we must have $e=r+1$. Clearly $t=n-r$ and as, by Theorem 3.2, $c-1 \leq t \leq c$, it follows that $n-c \leq r \leq n-c+1$. As $e=r+1$ and $e \leq n-c+1$ we then must have

$$
r=n-c \text { and } e=n-c+1 \text {. }
$$

Notice then that $c=n-r=t=1+r(r-1) / 2$. If on the other hand $t \leq r(r-1) / 2$ then, by Theorem 3.2 (b), we have $c \leq 1+r(r-1) / 2$. Thus $1+r(r-1) / 2$ is an upperbound for the powerful class of a powerfully nilpotent Lie ring of rank $r$ that has maximal tail.

Lemma 3.3 Suppose $L$ is a powerfully nilpotent Lie ring of p-power order that has maximal tail, is of rank $r$ and has powerful class $c=1+r(r-1) / 2$. Then L has tail of length $t=c$.

Proof We argue by contradiction and suppose $t \neq c$. By Theorem 3.2 (b), we then have $t=c-1=r(r-1) / 2$. Then $e \leq n-c+1=t+r-(c-1)=r$. By Theorem 3.2 (a), the only way of getting tail of length $t=1+2+\cdots+(r-1)$ is if $\operatorname{rank}(L)=r, \operatorname{rank}(p L)=r-1, \ldots, \operatorname{rank}\left(p^{r-1} L\right)=1$.

By Theorem 2.5 and Lemma 2.7 we get a powerfully central chain

$$
\begin{aligned}
& L \quad=J_{(0,1)} \quad \geq \cdots \geq J_{(0, r)} \quad=p L \\
& p L \quad=J_{(1,1)} \quad \geq \cdots \geq J_{(1, r-1)}=p^{2} L \\
& p^{r-2} L=J_{(r-2,1)} \geq \quad J_{(r-2,2)}=p^{r-1} L .
\end{aligned}
$$


Notice that $\left[p^{r-2} L, L\right]=p\left(p^{r-1} L\right)=p^{r} L=\{0\}$. We can thus replace $p^{r-1} L$ by $\{0\}$ and still have a powerfully central chain. That shows that the powerful class of $L$ is a most the length of chain namely $(r-1)+(r-2)+\cdots+1=r(r-1) / 2$ contradicting the assumption that the powerful class is $1+r(r-1) / 2$.

Definition Let $L$ be a powerfully nilpotent Lie ring with maximal tail. We say that $L$ has maximal powerful class if the powerful class is $c=1+r(r-1) / 2$.

Remark We know from Lemma 3.3 that if $L$ is a powerfully nilpotent Lie ring of rank $r$ which has maximal powerful class, then $t=c=1+r(r-1) / 2$ and from the analysis above [see the proof of Theorem 3.2 (c)] we know that as an abelian group we can write

$$
L=\mathbb{Z} a_{0} \oplus \cdots \oplus \mathbb{Z} a_{r-1}
$$

where the $\left\{o\left(a_{0}\right), \ldots, o\left(a_{r-1}\right)\right\}=\left\{p, p^{2}, \ldots, p^{r-1}, p^{r+1}\right\}$. For the time being suppose we have chosen $a_{0}, a_{1}, \ldots, a_{r-1}$ such that these give us a powerfully central chain like in Proposition 2.5. That is we have

$$
\begin{aligned}
J_{0} & =L=\mathbb{Z} a_{0}+\cdots+\mathbb{Z} a_{r-1}, J_{1}=\mathbb{Z} a_{1}+\cdots+\mathbb{Z} a_{r-1}+p L, \ldots J_{r-1} \\
& =\mathbb{Z} a_{r-1}+p L, J_{r}=p L
\end{aligned}
$$

where

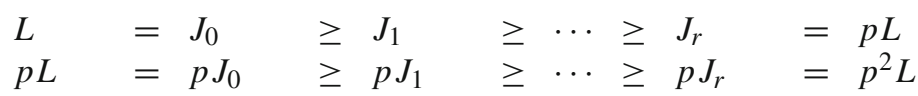

$$
\begin{aligned}
& p^{r-2} L=p^{r-2} J_{0} \geq p^{r-2} J_{1} \geq \cdots \geq p^{r-2} J_{r}=p^{r-1} L
\end{aligned}
$$

with $p^{r-1} L$ is of rank 1 and thus contained in $Z(L)$. We also know from Lemma 2.7 that if we omit repetitions then the rows give us subchains of lengths $r, r-1, \ldots, 2$ and that we still get a powerfully central chain if we omit the 2 nd term in each line. That would give us a powerfully central chain of length $(r-1)+(r-2)+\cdots+1+1=$ $1+r(r-1) / 2=c$ (adding $\left.p^{r-1} L>\{0\}\right)$. As this is the powerful class there can't be any strict subchain that is powerfully central. In particular

$$
\begin{aligned}
{\left[J_{0}, L\right]=} & {\left[J_{1}, L\right] \leq p J_{2},\left[J_{2}, L\right] \leq p J_{3}, \ldots\left[J_{r-2}, L\right] \leq p J_{r-1} . } \\
& {\left[J_{0}, L\right] \not \leq J_{3},\left[J_{2}, L\right] \not \leq p J_{4}, \ldots\left[J_{r-2}, L\right] \not \leq p J_{r} . }
\end{aligned}
$$

Notice that it follows from this that none of $a_{2}, \ldots, a_{r-1}$ can have order $p$, as if $o\left(a_{j}\right)=p$ then $\left[J_{j-1}, L\right] \leq p J_{j}=p J_{j+1}$ giving us a strict subchain that is powerfully central. It also follows that none of $a_{2}, \ldots, a_{r-1}$ can have order $p^{r+1}$. To see this we observe that if $a_{j}$ is of order $p^{r+1}$ for some $j \geq 2$ then by (2) there are some $0 \leq k<l \leq r-1$ such that

$$
\left[a_{k}, a_{l}\right]=p \alpha a_{j}+p u
$$


for some $u \in J_{j+1}$ and where $p \nmid \chi \alpha$. But then the right hand side has order $p^{r}$ whereas the left hand side has order at most $p^{r-1}$ (as one of $a_{k}, a_{l}$ has order at most $p^{r-1}$ ). By this contradiction we see that none of $a_{2}, \ldots, a_{r-1}$ can have order $p^{r+1}$. Thus $\left\{o\left(a_{0}\right), o\left(a_{1}\right)\right\}=\left\{p, p^{r+1}\right\}$ and $\left\{o\left(a_{2}\right), \ldots, o\left(a_{r-1}\right)\right\}=\left\{p^{2}, \ldots, p^{r-1}\right\}$.

Now notice that $\mathbb{Z} p a_{2}+p J_{3}=\left[J_{1}, L\right]+p J_{3}=\mathbb{Z}\left[a_{0}, a_{1}\right]+p J_{3}$. In particular $\left[a_{0}, a_{1}\right]=p \alpha a_{2}+p u$ with $u \in J_{3}$ and where $\alpha$ is not divisible by $p$. As $p\left[a_{0}, a_{1}\right]=0$ we then can't have that $p a_{2}$ has order greater than $p$ and thus $a_{2}$ must have order exactly $p^{2}$. Next we use

$$
\mathbb{Z} p a_{3}+p J_{4}=\left[J_{2}, L\right]+p J_{4}=\mathbb{Z}\left[a_{2}, a_{0}\right]+\mathbb{Z}\left[a_{2}, a_{1}\right]+p J_{4}
$$

As $p^{2}\left[a_{0}, a_{2}\right]=p^{2}\left[a_{1}, a_{2}\right]=0$ we can't have that the order of $p a_{3}$ is greater than $p^{2}$ and thus $o\left(a_{3}\right)=p^{3}$. Continuing inductively in this manner we see that we must have

$$
o\left(a_{2}\right)=p^{2}, o\left(a_{3}\right)=p^{3}, \ldots, o\left(a_{r-1}\right)=p^{r-1} .
$$

As $\left[a_{1}, a_{0}\right]=-\left[a_{0}, a_{1}\right]$ we can without loss of generality assume that $o\left(a_{1}\right)=p$ and $o\left(a_{0}\right)=p^{r+1}$ and that we then still have that modulo $p^{2} L$ that $J_{0}>J_{1}>J_{2}>\cdots>$ $J_{r}>\{0\}$ is powerfully central. We thus get:

Lemma 3.4 Let $L$ be a powerfully nilpotent Lie ring of rank $r$ and maximal powerful class. We can pick our generators $a_{0}, a_{1}, \ldots, a_{r-1}$ such that o( $\left.a_{1}\right)=$ $p, \ldots, o\left(a_{r-1}\right)=p^{r-1}$ and $o\left(a_{0}\right)=p^{r+1}$.

Remark In fact our proof also shows that we must have $o\left(a_{j}\right)=p^{j}$ for $2 \leq j \leq r-1$. For the following we will denote $a_{0}$ by $x$.

Lemma 3.5 We have $\left[a_{i}, x\right] \in p J_{i+1} \backslash p J_{i+2}$ for $i=1, \ldots, r-2$ and $\left[a_{r-1}, x\right] \in$ $p^{2} J_{0} \backslash p^{2} J_{1}$.

Proof Let $1 \leq i \leq r-2$. We have seen above that

$$
\mathbb{Z}\left[a_{i}, a_{1}\right]+\cdots+\mathbb{Z}\left[a_{i}, a_{r-1}\right]+\mathbb{Z}\left[a_{i}, x\right]+p J_{i+2}=\mathbb{Z} p a_{i+1}+p J_{i+2} .
$$

As the orders of $\left[a_{i}, a_{1}\right], \ldots,\left[a_{i}, a_{i-1}\right]$ divide $p^{i-1}$ whereas the order of $p a_{i+1}$ is $p^{i}$ it is easy to see that we must have that $\left[a_{i}, a_{1}\right], \ldots,\left[a_{i}, a_{i-1}\right] \in p J_{i+2}$. Also $\left[a_{i}, a_{j}\right] \in p J_{j+1} \leq p J_{i+2}$ for $j=i+1, \ldots, r-1$. Hence

$$
\mathbb{Z}\left[a_{i}, x\right]+p J_{i+2}=\mathbb{Z} p a_{i+1}+p J_{i+2}
$$

from which it follows that $\left[a_{i}, x\right] \in p J_{i+1} \backslash p J_{i+2}$. Similarly

$$
\mathbb{Z}\left[a_{r-1}, a_{1}\right]+\cdots+\mathbb{Z}\left[a_{r-1}, a_{r-2}\right]+\mathbb{Z}\left[a_{r-1}, x\right]+p^{2} J_{1}=\mathbb{Z} p^{2} x+p^{2} J_{1} .
$$

As $p^{2} x$ has order $p^{r}$ whereas $\left[a_{r-1}, a_{1}\right], \ldots,\left[a_{r-1}, a_{r-2}\right]$ have orders dividing $p^{r-1}$ we must have that $\left[a_{r-1}, a_{1}\right], \ldots,\left[a_{r-1}, a_{r-2}\right] \in p^{2} J_{1}$ and that

$$
\mathbb{Z}\left[a_{r-1}, x\right]+p^{2} J_{1}=\mathbb{Z} p^{2} x+p^{2} J_{1}
$$


from which it follows that $\left[a_{r-1}, x\right] \in p^{2} J_{0} \backslash p^{2} J_{1}$.

The arguments above also show that modulo $p\left(\mathbb{Z} p a_{2}+\cdots+\mathbb{Z} p a_{r-1}+\mathbb{Z} p^{2} x\right)$, we have

$$
\left[a_{1}, x\right]=p a_{2}, \ldots,\left[a_{r-2}, x\right]=p a_{r-1},\left[a_{r-1}, x\right]=p^{2} x
$$

It follows that the linear map

$$
\mathbb{Z} a_{1}+\cdots+\mathbb{Z} a_{r-1} \rightarrow \mathbb{Z} p a_{2}+\cdots+\mathbb{Z} p a_{r-1}+\mathbb{Z} p^{2} x, u \mapsto[u, x]
$$

is surjective and thus bijective as the two submodules are of the same order, namely $p^{1+2+\cdots+r-1}$. There thus exist unique $b_{r-1}, b_{r-2}, \ldots, b_{1} \in \mathbb{Z} a_{1}+\cdots+\mathbb{Z} a_{r-1}$ such that

$$
\left[b_{r-1}, x\right]=p^{2} x,\left[b_{r-2}, x\right]=p b_{r-1}, \ldots,\left[b_{1}, x\right]=p b_{2} .
$$

As the right Lie multiplication gives us a bijective map, $b_{1}, \ldots, b_{r-1}$ must be a basis for $\mathbb{Z} a_{1}+\cdots+\mathbb{Z} a_{r-1}$. In particular $\left\{o\left(b_{1}\right), \ldots, o\left(b_{r-1}\right)\right\}=\left\{p, p^{2}, \ldots, p^{r-1}\right\}$. Now we see from (3) that $o\left(b_{1}\right)\left|p, o\left(b_{2}\right)\right| p^{2}, \ldots, o\left(b_{r-1}\right) \mid p^{r-1}$ and we conclude that we must have $o\left(b_{1}\right)=p, \ldots, o\left(b_{r-1}\right)=p^{r-1}$. This proves the part (a) of our main structure theorem for Lie rings with maximal powerful class.

Theorem 3.6 Let $L$ be a powerfully nilpotent Lie ring of p-power order and rank $r$ that has maximal powerful class $1+r(r-1) / 2$. There exist generators $a_{1}, \ldots, a_{r-1}, x$ such that we get a direct sum of $\mathbb{Z}$-modules

$$
\mathbb{Z} x+\mathbb{Z} a_{1}+\cdots+\mathbb{Z} a_{r-1}
$$

where $o\left(a_{i}\right)=p^{i}$ and $o(x)=p^{r+1}$ and where for $J_{0}=L=\mathbb{Z} x+\mathbb{Z} a_{1}+\cdots+\mathbb{Z} a_{r-1}$, $J_{1}=\mathbb{Z} p x+\mathbb{Z} a_{1}+\cdots+\mathbb{Z} a_{r-1}, J_{2}=\mathbb{Z} p x+\mathbb{Z} p a_{1}+\mathbb{Z} a_{2}+\cdots+\mathbb{Z} a_{r-1}, \cdots$, $J_{r}=\mathbb{Z} p x+\mathbb{Z} p a_{1}+\cdots+\mathbb{Z} p a_{r-1}$ the chain

$$
\begin{aligned}
& L=J_{0}>J_{2}>\cdots>J_{r}=p L \\
& p L \quad=p J_{0}>p J_{3}>\cdots \quad>p J_{r}=p^{2} L \\
& p^{r-2} L=p^{r-2} J_{0} \quad>p^{r-2} J_{r} \quad>\{0\}
\end{aligned}
$$

is powerfully central of length $1+r(r-1) / 2$. Furthermore we can choose our generators such that

(a) $\left[a_{1}, x\right]=p a_{2},\left[a_{2}, x\right]=p a_{3}, \ldots,\left[a_{r-2}, x\right]=p a_{r-1},\left[a_{r-1}, x\right]=p^{2} x$.

(b) $H=\Omega_{r}(L)=\mathbb{Z} p x+\mathbb{Z} a_{1}+\cdots+\mathbb{Z} a_{r-1}$ is powerfully embedded in $L$ and strongly powerful.

(c) $p^{r-1} L \leq Z(L)$. 
Proof It only remains to prove (b) and (c). Part (c) follows from $p^{r-1} L=p^{r-1} \mathbb{Z} x$ being of rank 1 and Lemma 2.6. To see why (b) holds notice first that for $1 \leq i<j \leq$ $r-1$ we have

$$
\left[a_{i}, a_{j}\right]=p^{2} \alpha x+p^{2} \alpha_{1} a_{1}+\cdots+p^{2} \alpha_{j} a_{j}+p \alpha_{j+1} a_{j+1}+\cdots+p \alpha_{r-1} a_{r-1} .
$$

As $p^{r-2}\left[a_{i}, a_{j}\right]=0$ we then must have that $p$ divides $\alpha$ and as $p^{j-1}\left[a_{i}, a_{j}\right]=0$ we must have that $p$ divides $\alpha_{j+1}, \ldots, a_{r-1}$. Thus $\left[a_{i}, a_{j}\right] \leq p^{2} H$. Furthermore $\left[a_{i}, p x\right]=p^{2} a_{i+1}$ when $1 \leq i \leq r-2$ whereas $\left[a_{r-1}, p x\right]=p^{3} x$. Hence $[H, H] \leq$ $p^{2} H$ and $H$ is strongly powerful. Also $\left[a_{i}, x\right]=p a_{i+1}$ for $1 \leq i \leq r-2$ and $\left[a_{r-1}, x\right]=p^{2} x$ and thus $[H, L] \leq p H$ that shows that $H$ is powerfully embedded in $L$.

Remark The linear map induced by the Lie multiplication by $x$ from the right induces a surjective map

$$
\hat{Z}_{i}(L) / \hat{Z}_{i-1}(L) \rightarrow p \hat{Z}_{i-1}(L) / p \hat{Z}_{i-2}(L)
$$

for $2 \leq i \leq c$. One could think of this property as corresponding to the property of being a uniform element in the theory of $p$-groups of maximal class. Notice also that Lemma 2.1 tells us that the class of $H$ is at most $r$ that is small compared to the class of $L$.

Having analysed the structure of powerfully nilpotent Lie rings of maximal powerful class, we show that for each $r$ there exists such a Lie ring.

Let $r$ be a positive integer and let $p$ be a prime. Consider a $\mathbb{Z}$-module

$$
L=\mathbb{Z} x \oplus \mathbb{Z} a_{1} \oplus \cdots \oplus \mathbb{Z} a_{r-1}
$$

where $o\left(a_{i}\right)=p^{i}$ and $o(x)=p^{r+1}$. Define a right multiplication on $A=\mathbb{Z} a_{1} \oplus \cdots \oplus$ $\mathbb{Z} a_{r-1}$ by $x$ where this is the linear map induced by

$$
\left[a_{1}, x\right]=p a_{2},\left[a_{2}, x\right]=p a_{3}, \ldots,\left[a_{r-2}, x\right]=p a_{r-1},\left[a_{r-1}, x\right]=p^{2} x .
$$

With a slight abuse of notation we will also denote this linear map by $x$. Notice that the image of $x$ is $p A+\mathbb{Z} p^{2} x$ that has the same order as $A$, namely $p p^{2} \cdots p^{r-1}$, and thus $x: A \rightarrow L$ is injective.

Now consider an alternating product on $A$ such that $[A, A] \subseteq B$ where $B=$ $\mathbb{Z} a_{1}+\cdots+\mathbb{Z} a_{r-2}$. Our aim is to find such an alternating product that together with the right multiplication of $x$ will give us a Lie ring.

Proposition 3.7 There exists a unique alternating product on A with the following properties
(1) $[A, A] \subseteq B$;
(2) $[[a, b], x]=[[a, x], b]+[a,[b, x]]$ for all $a, b \in A$.

Furthermore this alternating product of A together with the right multiplication by $x$ gives $L$ the structure of a Lie ring. Also $[A, A] \subseteq p^{2} B$. 
Proof Notice that the linear map $x$ induces a bijection from $B$ to $p A$. Thus for any $u \in p A$ there exists a unique $v \in B$ such that $[v, x]=u$. We will be making use of this property. We will first establish (1) and (2) as well as $[A, A] \subseteq p^{2} B$. Let $1 \leq i<j \leq r-1$. We define $\left[a_{i}, a_{j}\right]$ by reverse induction on $3 \leq i+j \leq 2 r-3$. We will show that there is a unique $u \in B$ such that $p^{i} u=0$ and

$$
[u, x]=\left[\left[a_{i}, x\right], a_{j}\right]+\left[a_{i},\left[a_{j}, x\right]\right]
$$

and we define $\left[a_{i}, a_{j}\right]=u$. We will also see that in fact $u \in p^{2} B$.

For the induction basis consider $i=r-2$ and $j=r-1$. Then

$$
\left[\left[a_{i}, x\right], a_{j}\right]+\left[a_{i},\left[a_{j}, x\right]\right]=p\left[a_{r-1}, a_{r-1}\right]+\left[a_{r-2}, p^{2} x\right]=p^{3} a_{r-1}
$$

Using the fact that $x: B \rightarrow p A$ is injective we know that $u=p^{2} a_{r-2}$ is the unique element in $B$ such that $[u, x]=p^{3} a_{r-1}$. Notice that $u \in p^{2} B$ and that $p^{r-2} u=0$. We define $\left[a_{r-2}, a_{r-1}\right]=p^{2} a_{r-2}$.

For the induction step suppose $3 \leq s=i+j<2 r-3$ and that our inductive claim and definition holds whenever $s<i+j \leq 2 r-3$. If $j=r-1$ then let

$$
v=\left[p a_{i+1}, a_{r-1}\right]+\left[a_{i}, p^{2} x\right]=p\left[a_{i+1}, a_{r-1}\right]+p^{3} a_{i+1} .
$$

By the induction hypothesis we know that $\left[a_{i+1}, a_{r-1}\right] \in p^{2} B$ and thus $v \in p^{3} A$. As before we know that there is a unique $u \in p^{2} B$ such that $[u, x]=v$. We let $\left[a_{i}, a_{j}\right]=$ $u$. Notice also that by the induction hypothesis we know that $p^{i+1}\left[a_{i+1}, a_{r-1}\right]=0$. Thus it follows that $p^{i} v=0$. By the property of the function $x$ we know that we also have $p^{i} u=0$.

Now consider the case when $j<r-1$. Let

$$
v=\left[p a_{i+1}, a_{j}\right]+\left[a_{i}, p a_{j+1}\right]=p\left[a_{i+1}, a_{j}\right]+p\left[a_{i}, a_{j+1}\right] .
$$

By the induction hypothesis we know that $v \in p^{3} B$ and we know there is a unique $u \in p^{2} B$ such that $[u, x]=v$. We let $\left[a_{i}, a_{j}\right]=u$. Again we know by the induction hypothesis that $p^{i+1}\left[a_{i+1}, a_{j}\right]=p^{i+1}\left[a_{i}, a_{j+1}\right]=0$ and thus $p^{i} v=0$. Thus also $p^{i} u=0$ by the properties of the linear map $x$. This finishes the inductive proof.

Having established that there is this unique alternating product on $A$ such that (1) and (2) hold and that furthermore $[A, A] \subseteq p^{2} B$ we extend this into an alternating product on $L$ using the right multiplication on $A$ by $x$. Notice that by (2) we know that $x$ acts as derivation on $A$. In order to show that the Jacobi identity $J(x, y, z)=0$ holds where

$$
J(x, y, z)=[[x, y], z]+[[y, z], x]+[[z, x], y]
$$

it then clearly suffices to show that $J\left(a_{i}, a_{j}, a_{k}\right)=0$ whenever $1 \leq i<j<k \leq r-1$. We prove this by reverse induction on $6 \leq i+j+k \leq 3(r-2)$. By the first part of 
the proof we know that $J\left(a_{i}, a_{j}, a_{k}\right) \in p^{2} B$. As $x: B \rightarrow p A$ is injective it suffices to show that $\left[J\left(a_{i}, a_{j}, a_{k}\right), x\right]=0$. For the induction basis notice that

$$
\begin{aligned}
{\left[J\left(a_{r-3}, a_{r-2}, a_{r-1}\right), x\right]=} & p J\left(a_{r-2}, a_{r-2}, a_{r-1}\right)+p J\left(a_{r-3}, a_{r-1}, a_{r-1}\right) \\
& +p^{2} J\left(a_{r-3}, a_{r-2}, x\right) \\
= & p^{2} J\left(a_{r-3}, a_{r-2}, x\right) \\
= & 0
\end{aligned}
$$

where the last equality follows from (2).

For the induction step, suppose $6 \leq i+j+k=s<3(r-2)$ and that the Jacobi identity holds whenever $s<i+j+k \leq 3(r-2)$. If $k=r-1$ then

$$
\begin{aligned}
{\left[J\left(a_{i}, a_{j}, a_{k}\right), x\right] } & =p J\left(a_{i+1}, a_{j}, a_{k}\right)+p J\left(a_{i}, a_{j+1}, a_{k}\right)+p^{2} J\left(a_{i}, a_{j}, x\right) \\
& =p^{2} J\left(a_{i}, a_{j}, x\right) \\
& =0
\end{aligned}
$$

where again the last equality follows from (2). Thus suppose that $k<r-1$. Then

$$
\left[J\left(a_{i}, a_{j}, a_{k}\right), x\right]=p J\left(a_{i+1}, a_{j}, a_{k}\right)+p J\left(a_{i}, a_{j+1}, a_{k}\right)+p J\left(a_{i}, a_{j}, a_{k+1}\right)=0 .
$$

This finishes the inductive proof of the Jacobi identity and thus the lemma.

We have thus shown the existence of a Lie ring $L=\mathbb{Z} a_{1}+\cdots+\mathbb{Z} a_{r-1}+\mathbb{Z} x$ where

$$
\left[a_{1}, x\right]=p a_{2}, \ldots,\left[a_{r-2}, x\right]=p a_{r-1}, \quad \text { and }\left[a_{r-1}, x\right]=p^{2} x
$$

and where furthermore $[A, A] \leq p^{2} B$. Notice that $[L, L] \leq p L$ and thus one sees inductively that $L^{r} \leq p^{r-1} L=\mathbb{Z} p^{r-1} x \leq Z(L)$. This implies that the nilpotency class of $L$ is at most $r$. In fact $\left[a_{1}, r-1 x\right]=p\left[a_{2}, r-2 x\right]=\cdots=p^{r-2}\left[a_{r-1}, x\right]=$ $p^{r} x \neq 0$ and the class is thus exactly $r$.

Theorem 3.8 The Lie ring $L$ is a powerfully nilpotent Lie ring with maximal tail.

Proof Let $L_{0}=L, L_{1}=\mathbb{Z} p x+\mathbb{Z} a_{1}+\mathbb{Z} a_{2}+\cdots+\mathbb{Z} a_{r-1}, L_{2}=\mathbb{Z} p x+\mathbb{Z} p a_{1}+\mathbb{Z} a_{2}+$ $\mathbb{Z} a_{3}+\cdots+\mathbb{Z} a_{r-1}, \cdots, L_{r}=p L$ and consider the descending chain of subrings

$$
\begin{gathered}
L=L_{0}>L_{1}>\cdots>L_{r}=p L \\
p L=p L_{0} \geq p L_{1} \geq \cdots \geq p L_{r}=p^{2} L \\
\vdots \\
p^{r} L=p^{r} L_{0} \geq p^{r} L_{1} \geq \cdots \geq p^{r} L_{r}=p^{r+1} L=\{0\} .
\end{gathered}
$$

As $[A, A] \leq p^{2} B$ and $\left[L_{i}, x\right] \leq p L_{i+1}$, it follows that this chain is powerfully central and thus $L$ is powerfully nilpotent. In order to determine the upper powerfully central series we make again use of $[A, A] \leq p^{2} B$ as well as the fact that 
$x: A \rightarrow p A+\mathbb{Z} p^{2} x$ is injective. From this one sees readily that the following ascending sequences are $\left(\hat{Z}_{i}(L)\right)$ and $\left.\left(p \hat{Z}_{i}(L)\right)\right)$ :

$$
\begin{aligned}
& \left\langle p^{r-1} x\right\rangle \\
& \left\langle p^{r} x\right\rangle \\
& \\
& <\left\langle p^{r-2} x, p^{r-2} a_{r-1}\right\rangle \\
& <\left\langle p^{r-1} x\right\rangle \\
& <\left\langle p^{r-2} x, p^{r-3} a_{r-1}\right\rangle \quad \\
& <\left\langle p^{r-1} x, p^{r-2} a_{r-1}\right\rangle \quad<\left\langle p^{r-3} x, p^{r-3} a_{r-2}, p^{r-3} a_{r-1}\right\rangle \\
&
\end{aligned}
$$

$<\left\langle p x, \ldots, p a_{r-2}, a_{r-1}\right\rangle \quad<\left\langle p x, \ldots, p a_{r-3}, a_{r-2}, a_{r-1}\right\rangle \quad<\cdots<\left\langle p x, p a_{1}, a_{2}, \ldots, a_{r-1}\right\rangle<L$ $<\left\langle p^{2} x, \ldots, p^{2} a_{r-2}, p a_{r-1}\right\rangle<\left\langle p^{2} x, \ldots, p^{2} a_{r-3}, p a_{r-2}, p a_{r-1}\right\rangle<\cdots<\left\langle p^{2} x, p a_{2}, \ldots, p a_{r-1}<p L\right.$

All the factors of the chain $\left(p \hat{Z}_{i}(L)\right)$ are of order $p$ and thus $L$ has maximal tail of length $1+(1+2+\cdots+r-1)=1+r(r-1) / 2$.

\section{Powerfully nilpotent groups with maximal powerful class}

In this section we want to prove results on powerfully nilpotent groups with maximal powerful class that are analogous to those we have obtained for the powerfully nilpotent Lie rings.

Let $p$ be a prime. We will make use of the Lazard 1-1 correspondence between all finite $p$-groups of nilpotency class at most $p-1$ and all finite Lie rings of class at most $p-1$ whose order is a power of $p$.

For a full account of the Lazard correspondence see for example [3]. Let us recall briefly the setting. Let $L$ be a Lie ring of order $p^{n}$ and nilpotency class $m<p$. The corresponding group $G=\left\{e^{a}: a \in L\right\}$ is then a finite group of the same order and nilpotency class consisting of formal expressions

$$
e^{a}=1+a+\frac{a}{2 !}+\cdots+\frac{a^{m}}{m !} .
$$

(To be more precise, one can view this as an expression within $1+E$ where $E$ is the associative envelping algebra of $L$.) The structure of $G$ is derived from the structure of $L$, via the Baker-Hausdorff formula. Thus

$$
e^{a} \cdot e^{b}=e^{l(a, b)}
$$

where $l(x, y)$ is a word in the free Lie algebra over $\mathbb{Q}$ of class $m$ provided by the Lazard correpondence and $l(a, b)$ is the value of $l(x, y)$ in $L$. In the following we will use the bracket notion both for group commutators and for the Lie product as there will be no ambiguity regarding this. Under the Lazard correspondence, one has in particular 


$$
\begin{aligned}
{\left[e^{a}, e^{b}\right] } & =e^{B(a, b)} \\
e^{[a, b]} & =C\left(e^{a}, e^{b}\right)
\end{aligned}
$$

where $B(a, b) \in\langle a, b\rangle^{\prime}$ and $C\left(e^{a}, e^{b}\right) \in\left\langle e^{a}, e^{b}\right\rangle^{\prime}$.

Under the Lazard correspondence, sub Lie rings of $L$ correspond to subgroups of $G$ and ideals of $L$ correspond to normal subgroups of $G$. Notice also that if $M$ is a subring of $L$ and $H=e^{M}$ is the corresponding subgroup of $G$, then the subgroup corresponding to the subring $p M$ is $e^{p M}=\left\{h^{p}: h \in H\right\}=H^{p}$.

Let $M, N$ be subrings of $L$. Notice that

$$
[M, L] \leq p N \quad \text { iff }\left[e^{M}, G\right] \leq\left(e^{N}\right)^{p}
$$

In particular it follows that $L$ is powerfully nilpotent with a powerfully central series

$$
\{0\}=L_{0} \leq L_{1} \leq \cdots \leq L_{n}=L
$$

if and only if $G$ is powerfully nilpotent with a powerfully central series

$$
\{1\}=e^{L_{0}} \leq e^{L_{1}} \leq \cdots \leq e^{L_{n}}=G .
$$

The former is a fastest ascending series if and only if the latter is. Thus we have

$$
\hat{Z}_{i}(G)=e^{\hat{Z}_{i}(L)}, \quad \text { for } i \in \mathbb{N} \text {. }
$$

Notice also that it follows that $L$ has maximal tail if and only if $G$ has maximal tail and that the two tails have then the same length.

In the previous section we constructed a powerfully nilpotent Lie ring of $p$-power order and rank $r$ that is of maximal powerful class and saw that the real class of $L$ was $r$. In the case when $p>r$, we can thus use the Lazard Correspondence to get a corresponding example $G=e^{L}$ that is a powerfully nilpotent group of maximal powerful class.

Theorem 4.1 Let $r$ be a positive integer and $p>r$ a prime. There exists a powerfully nilpotent $p$-group of rank $r$ that is of maximal powerful class.

We next move to the analogue of the main structure theorem for the powerfully nilpotent Lie rings of maximal powerful class. Let again $p>r$ and suppose that

$$
L=\mathbb{Z} x \oplus \mathbb{Z} a_{1} \oplus \cdots \oplus \mathbb{Z} a_{r-1}
$$

with $o(x)=p^{r+1}, o\left(a_{1}\right)=p, \ldots, o\left(a_{r-1}\right)=p^{r-1}$, is a powerfully nilpotent Lie ring of $p$-power order that is of rank $r$ and maximal powerful class. We let $G=e^{L}$ be the corresponding powerfully nilpotent $p$-group that is of maximal powerful class. Recall 
that $x$ has the property that Lie multiplication by $x$ on the right induces surjective maps

$$
\hat{Z}_{i}(L) / \hat{Z}_{i-1}(L) \rightarrow p \hat{Z}_{i-1}(L) / p \hat{Z}_{i-2}(L)
$$

It follows that the commutator operation with $e^{x}$ from the right induces surjective group homomorphisms

$$
\hat{Z}_{i}(G) / \hat{Z}_{i-1}(G) \rightarrow\left(\hat{Z}_{i-1}(G)\right)^{p} /\left(\hat{Z}_{i-2}(G)\right)^{p} .
$$

From this one sees that the commutator operation with $e^{x}$ from the right gives us a surjective map from $G=e^{L}$ to $\hat{Z}_{c-1}(G)^{p}=\left\langle e^{p^{2} x}, e^{p a_{1}}, \ldots, e^{p a_{r-1}}\right\rangle$. Let $y=e^{x}$, we then get $b_{1}, \ldots, b_{r-1} \in G$ such that

$$
\left[b_{r-1}, y\right]=y^{p^{2}},\left[b_{r-2}, y\right]=b_{r-1}^{p},\left[b_{r-3}, y\right]=b_{r-2}^{p}, \ldots,\left[b_{1}, y\right]=b_{2}^{p} .
$$

Similar analysis as in the Lie ring situation shows that $o\left(b_{1}\right)=p, \ldots, o\left(b_{r-1}\right)=p^{r-1}$ and that

$$
G=\langle y\rangle\left\langle b_{1}\right\rangle \cdots\left\langle b_{r-1}\right\rangle
$$

where $y, b_{1}, \ldots, b_{r-1}$ play an analogous role to $x, a_{1}, \ldots, a_{r-1}$ in the Lie ring situation. More precisely we get the following result.

Theorem 4.2 Let $G$ be a powerfully nilpotent $p$-group of rank $r$ where $p>r$ that has maximal powerful class $1+r(r-1) / 2$. There exist generators $b_{1}, \ldots, b_{r-1}, y$ such that $G$ is a product of the corresponding cyclic groups

$$
G=\langle y\rangle \cdot\left\langle b_{1}\right\rangle \cdots\left\langle b_{r-1}\right\rangle
$$

with $|G|=o(y) o\left(b_{1}\right) \cdots o\left(b_{r-1}\right)$, where $o\left(b_{i}\right)=p^{i}$ and $o(y)=p^{r+1}$ and where for $H_{0}=G=\langle y\rangle \cdot\left\langle b_{1}\right\rangle \cdots\left\langle b_{r-1}\right\rangle, H_{1}=\left\langle y^{p}\right\rangle\left\langle b_{1}\right\rangle \cdots\left\langle b_{r-1}\right\rangle, H_{2}=\left\langle y^{p}\right\rangle \cdot\left\langle b_{1}^{p}\right\rangle$. $\left\langle b_{2}\right\rangle \cdots\left\langle b_{r-1}\right\rangle, \cdots, H_{r}=G^{p}$, the chain

$$
\begin{aligned}
& G \quad=H_{0}>H_{2}>\cdots>H_{r}=G^{p} \\
& G^{p}=H_{0}^{p}>H_{3}^{p}>\cdots>H_{r}^{p}=G^{p^{2}} \\
& G^{p^{r-2}}=H_{0}^{p^{r-2}} \quad>H_{r}^{p^{r-2}} \quad>\{1\}
\end{aligned}
$$

is powerfully central of length $1+r(r-1) / 2$. Furthermore we can choose our generators such that

(a) $\left[b_{1}, y\right]=b_{2}^{p},\left[b_{2}, y\right]=b_{3}^{p}, \ldots\left[b_{r-2}, y\right]=b_{r-1}^{p},\left[b_{r-1}, y\right]=y^{p^{2}}$.

(b) $H=\Omega_{r}(G)=\left\langle y^{p}\right\rangle \cdot\left\langle b_{1}\right\rangle \cdots\left\langle b_{r-1}\right\rangle$ is powerfully embedded in $G$ and strongly powerful. 
(c) $G^{p^{r-1}} \leq Z(G)$.

Remark The fact that $H$ is strongly powerful of exponent $p^{r}$ implies that its powerful class is at most $r$ and thus small compared to the powerful class of $G$. The situation is thus analogous to the structure of $p$-groups of maximal class as we have a subgroup of index $p$ with relatively small powerful class and a 'uniform' element $y$.

Open problem Do Theorems 4.1 and 4.2 still hold when $p \leq r$ ?

Acknowledgements This paper originated from the first author's visit to the University of the Basque Country. He thanks the department of mathematics for their great hospitality during the visit and in particular G. A. Fernanández-Alcober and M. Noce for fruitful discussions.

Open Access This article is distributed under the terms of the Creative Commons Attribution 4.0 International License (http://creativecommons.org/licenses/by/4.0/), which permits unrestricted use, distribution, and reproduction in any medium, provided you give appropriate credit to the original author(s) and the source, provide a link to the Creative Commons license, and indicate if changes were made.

\section{References}

1. Fernández-Alcober, G.A.: An introduction to finite $p$-groups: regular $p$-groups and groups of maximal class. In: Mathemática Contemporânea, vol. 20. XVI Escola de Álgebra (Part 1), pp. 155-225 (2001)

2. Huppert, B.: Endliche Gruppen I, Die Grundlehren der Mathematishcen Wissenshaften, vol. 134. Springer, Berlin (1967)

3. Khukhro, E.I.: p-Automorphisms of Finite $p$-Groups, LMS Lecture Note Series 246. Cambridge University Press, Cambridge (1998)

4. Leedham-Green, C.R., McKay, S.: The Structure of Groups of Prime Power Order, LMS Monographs (New Series) 27. Oxford University Press, Oxford (2002)

5. Lubotzky, A., Mann, A.: Powerful p-groups. J. Algebra 105, 485-505 (1987)

6. Traustason, G., Williams, J.: Powerfully nilpotent groups. J. Algebra 522, 80-100 (2019)

7. Williams, J.: Omegas of agemos in powerful groups. Int. J. Group Theory (to appear)

Publisher's Note Springer Nature remains neutral with regard to jurisdictional claims in published maps and institutional affiliations. 